\title{
Unforced Rayleigh instability of an immersed liquid jet
}

\author{
Claudiu Patrascu, ${ }^{1, *}$, Alina Chipaila ${ }^{1}$, and Corneliu Balan $^{1}$ \\ ${ }^{1}$ UPB, Hydraulics Department, REOROM Group, 313 Splaiul Independentei sect. 6, Bucharest, Romania
}

\begin{abstract}
Motivated by the occurrence of the injection of liquids in various technical processes, we study the capillary instability of a liquid jet surrounded by another liquid. The study focuses on the natural developing Rayleigh instability, hence without an imposed perturbation. We also point out the influence of viscosity on the main drop diameter, resulted after jet breakup, and on the breakup length itself. Modifications brought by a decrease of the capillary nozzle are also emphasized for a particular case.
\end{abstract}

\section{Introduction}

The study of liquid jet breakup is motivated by practical applications such as ink-jet printing, DNA sampling or dosing consumer products [1-3]. Besides the applicative side of jet instability, one may find that liquid jets can be used to highlight the influence of physical macroscopic properties such as viscosity, density, interfacial tension or elasticity in interfacial phenomena.For example, the relaxation time of weakly elastic polymer solutions, can be determined via R.O.J.E.R, a device specifically created that makes use of jet dynamics and filament thinning in the vicinity of drop detachment $[4,5]$. The density contrast between an injected fluid and its surrounding medium is still a subject of interest, considering that when immersed, the force of gravity is reduced by a factor proportional to the density difference between the two fluids [6]. The injection of a fluid in a certain industrial process might be subject to many perturbations, therefor the breakup process will be dependent of the superposition of allexcited modes. This causes a delay or a growth in the time needed for a drop to form and detach. The common approach to control jet breakup is to introduce a specific perturbation and excite a certain mode, but this approach results in a costly process. The study of an unforced breakup of a liquid jet may offer insight and a spawning ground for an alternative controlling technique.

The present study is structured as follows. We study the natural occurring capillary instability of a liquid jet surrounded by sunflower oil with no imposed perturbation. We emphasize the influence of viscosity and nozzle radius on the main and satellite drop diameter and on the breakup length.

\section{Theoretical aspects of jet breakup}

A liquid emanating from a nozzle experiences instability which leads to the formation of droplets (Fig. 1-a). In the case of a jetting regime, the breakup process can lead to the formation of main drops and satellite droplets.
Usually the diameter of the main drop, $D_{d}$ is much greater than that of the satellite droplet, $D_{s}$. This is encountered whether the exterior medium is a gas or a liquid, provided that the liquids are immiscible. At a flow rate sufficiently high that ensures the convection of any perturbation that the interface might experience, the dynamics are governed by capillarity and the phenomenon is called Rayleigh instability. It represents a convective type of instability, where the growth rate is considered a complex number and the wave number real and positive. Suitable in this case is the temporal analysis applied to perturbations that grow uniformly in space. This was first carried out by Lord Rayleigh [7] which showed that the dispersion equation, relating the growth rate of disturbance and the wave number, exhibits a maximum, which lead him to conclude that the fastest growing mode is responsible for jet breakup. The effect of viscosity on the dispersion relation was emphasized by Chandrasekhar [8] and the effect of a surrounding medium by Tomotika [9] which has the following form:

$i \underline{\omega}=\sigma\left[1-\left(k R_{0}\right)^{2}\right] f\left(k R_{0}, \beta\right) / 2 R_{0} \eta_{2}$,

where $\underline{\omega}$ is the complex growth rate (quantifying the rate at which perturbations grow in time), $\sigma$ is interfacial tension, $k$ is the wave number, $R_{0}$ the inner radius of the nozzle, $\eta_{2}$ is the viscosity of the outer fluid and $f\left(k R_{0}, \beta\right)$ a function of the nondimensional wave number and the viscosity ratio, respectively. Fig1-b shows the prediction for the growth rate as a function of the nondimensional wave number for three different injected liquids. Having determined the maximum of a particular curve, one can predict the breakup length by [10]:

$L_{b}=c V / i \underline{\omega}_{M}$.

In Eq. (2) $V$ is the mean velocity of the liquid jet and $c$ a pre-factor. The increase of concentration results in an increase of the viscosity of the injected liquid and subsequently of the viscosity ratio. This produces a

* Corresponding author: claudiu.t.patrascu@gmail.com 

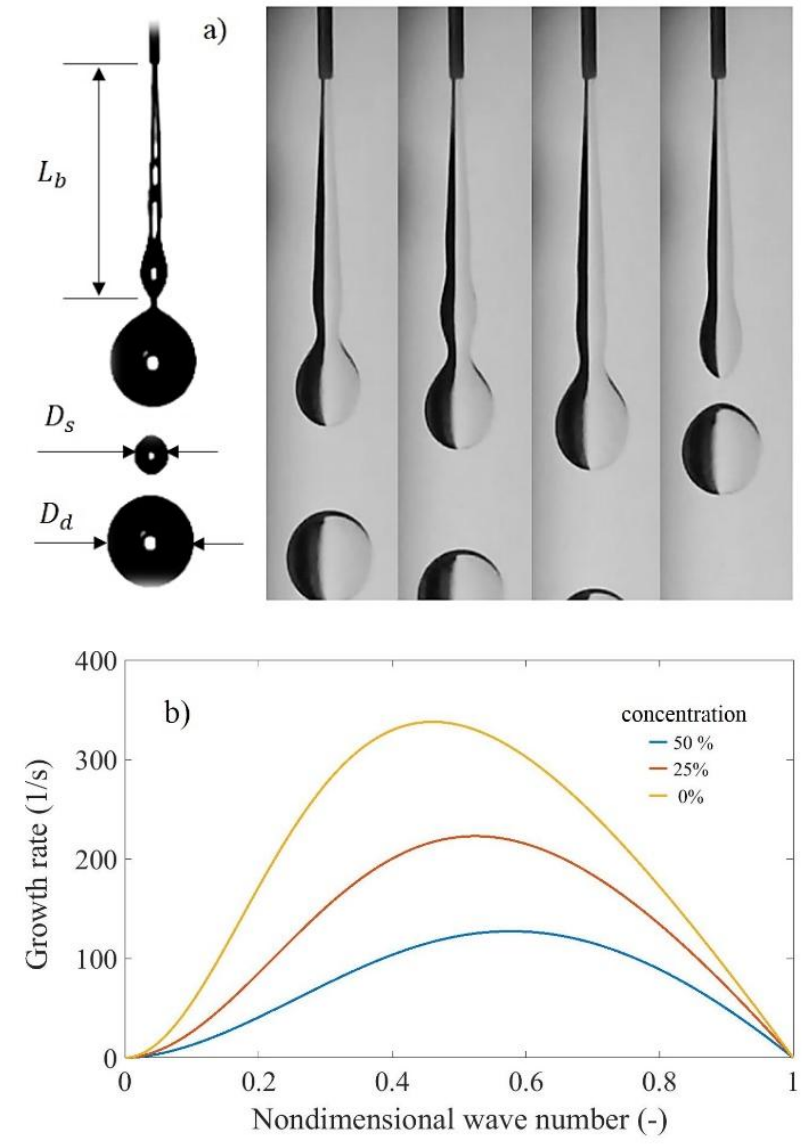

Fig. 1a) Schematic representation of the pursued parameters (left) and snapshots of the capillary instability of a liquid jet (right).The figure shows the breakup length, the main drop and the satellite droplet; b) Dispersion relation for three water and glycerin mixtures. The graph shows the dependence of the growth rate as a a function of the wave number and the influence of increasing the concentration of glycerin in water.

decrease in the maximum growth rate as predicted by Eq. (1). Decreasing the maximum value of the growth rate results in a decrease of the liquid intact length, if one expects the pre-factor to remain constant. Making the hypothesis that the fastest growing mode is responsible for breakup, we show that the pre-factor must change in order to capture the behaviour of the jet as viscosity is increased. The pre-factor $c$ is dependent on the radius of the nozzle and on the value of the initial amplitude $A_{0}$ introduced by the injection system:

$c=\ln \left(R_{0} / A_{0}\right)$.

Reference regarding this pre-factor can be found in [3].

\section{Experimental details}

The experimental setup consists of a glass tank filled with sunflower oil in which a water - glycerine mixture is injected with the use of a blunt tip nozzle. Images of the phenomenon were taken by a Nikon 1 J5 camera with $3840 \times 2160$ resolution at $400 \mathrm{fps}$. For the injection process we used a Harvard 33 syringe pump. Physical properties
Table 1. Values of the material properties at $25^{\circ} \mathrm{C}$ for the fluids subject to analysis.

\begin{tabular}{cccc}
\hline Sample & $\boldsymbol{\rho}\left[\mathbf{k g} / \mathbf{m}^{3}\right]$ & $\boldsymbol{\eta}[\boldsymbol{m P a s}]$ & $\boldsymbol{\sigma}[\mathbf{N} / \mathbf{m}]$ \\
\hline water & 1000 & 1 & 0.025 \\
\hline water $+25 \%$ glycerin & 1076 & 2.4 & 0.023 \\
\hline water + 50\% glycerin & 1143 & 7 & 0.021 \\
\hline sunflower oil & 925 & 55 & - \\
\hline
\end{tabular}

of the fluids in question were determined as follows: the viscosity was determined using a rotational rheometer in a standard oscillatory test, the density by a mass per volume method and interfacial tension via a pendant drop method. The values of the above-mentioned material properties are listed in Table 1 . We differentiate the injected fluidfrom the outer medium by sub-index 1 andsub-index 2,respectively. The viscosity and density of the injected fluid were increased by increasing the concentration of glycerine (in volume). The breakup of the liquid jet was not triggered in an imposed manner, but by a naturally occurring perturbation, caused by the injection process. Two characteristic parameters were measured, the breakup length, which represents the distant between the tip of the capillary nozzle and the point of drop detachment, and the diameters of the detached drops. For each parameter a series of 50 punctual measurements were performed.

\section{Results and comments}

A first comment can be made regarding the average value of the breakup length. Increasing the concentration of glycerine and therefor the viscosity and density of the injected fluid results in a decrease of the average value of

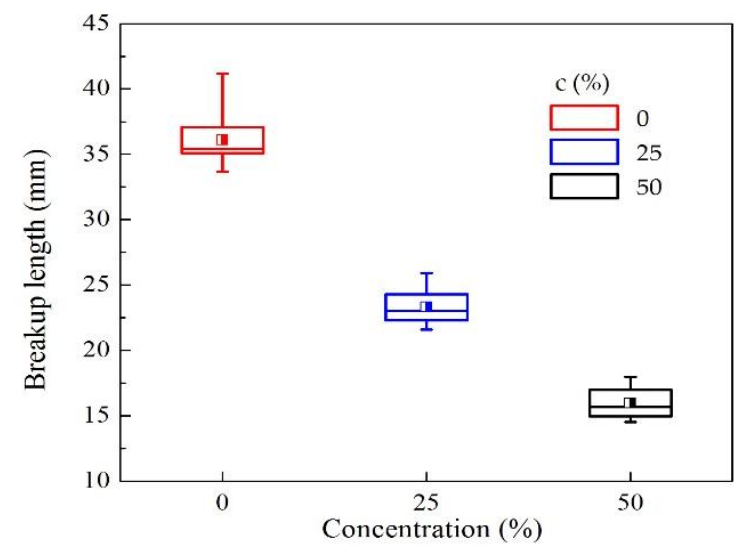

Fig. 2Average valuesof the breakup length as a function of concentration of glycerin in water at a flow rate of $11 \mathrm{ml} / \mathrm{min}$. The inner radius of the capillary tube is $0.3 \mathrm{~mm}$, the outer fluid being sunflower oil in all three cases. 

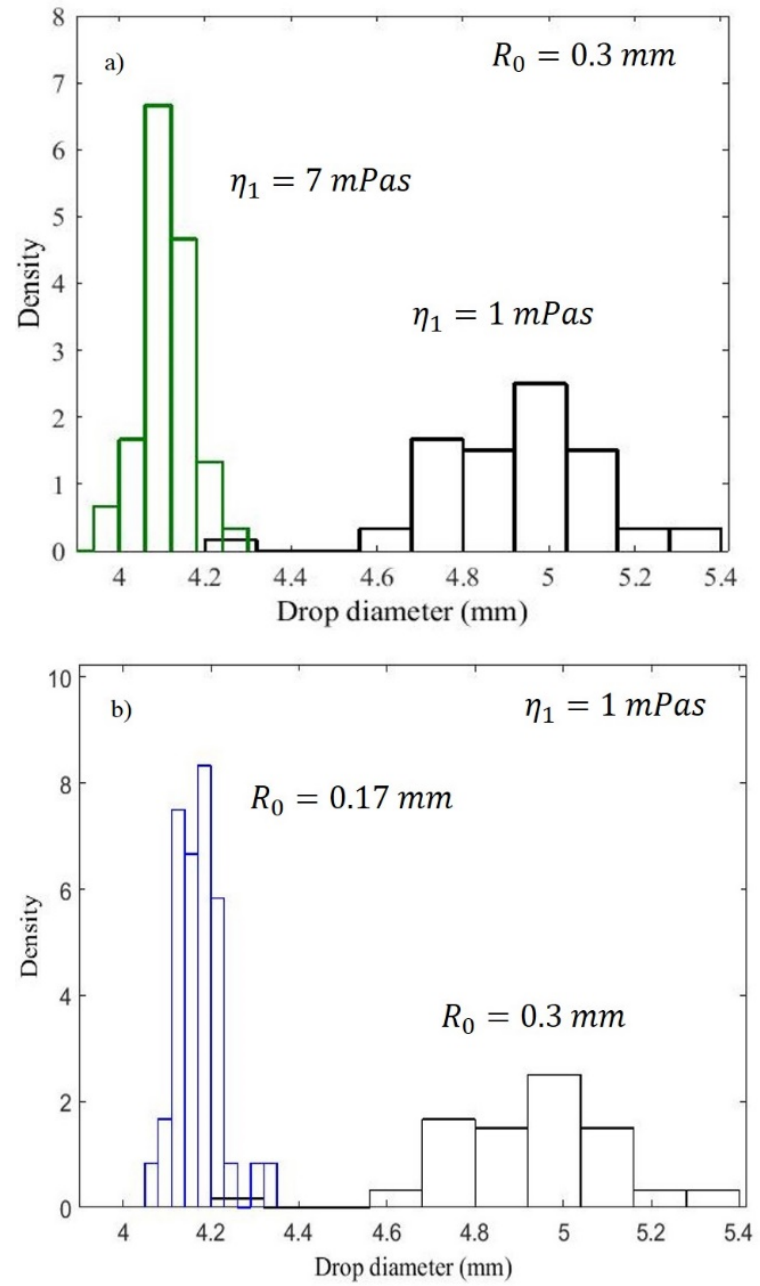

Fig. 3a) The influence of viscosity on the main drop diameter. A comparison between deionized water and a $50 \%$ glycerin in water mixture is presented, showing a decrease in both drop diameter and data dispersion; b) The influence of modifying the inner radius of the capillary tube on the main drop diameter in the case of deionized water. The same decrease in drop diameter and data dispersion is observed.

the breakup length (Fig 2). By inspection of Fig. 1-b and Eq. (2) we see that the maximum value presented by the growth rate decreases with viscosity, implying that the breakup length increases if one considers a constant value for the pre-factor. Under the hypothesis that the most unstable mode is responsible for breakup, the prefactor must decrease, thus the initial amplitude must increase, although it is common knowledge that viscosity has a damping role. The injection system might also excite multiple modes, a subject not treated here, that could alter the linear predictions of Eq. (2).

Next, we look at the diameter of the main drop in two particular cases. First, the viscosity of the injected fluid is increased from $1 \mathrm{mPas}$ to $7 \mathrm{mPas}$ (Fig. 3-a). The immediate effect of the latter is a decrease in the average value of the main drop diameter and also a decrease in data dispersion. The same effect is encountered if one modifies the inner radius of the nozzle. Both data dispersion and average value of the main diameter decrease (Fig. 3-b). A decrease in drop diameter with viscosity is seen in cases where the jetting regime is replaced by a dripping regime. Partially, this is due to the fact that interfacial tension decreases and density increases,causing the drop diameter to decrease.

For a deionized water in sunflower oil system, the satellite droplets can be very small in size compared to the main droplets. The data is dispersed, but the phenomenon still retains its periodicity when speaking of drop generation. The main drop is first generated followed by one or two smaller in size. When increasing viscosity of the injected fluid, the ratio between two consecutive drop diameters decreases(Fig. 4). Usually the smaller drop is referred to as a satellite drop, but in this case the latter is comparable in size with the main drop. This suggests that increasing the viscosity even further would totally eliminate the occurrence of satellite droplets. Further investigations are required to establish if this is indeed the case.

It is worth mentioning that above a certain critical concentration of glycerine in water the breakup length starts to increase, despite the variation that one would expect by prospecting Fig. 2. For example, a water glycerine mixture with the same viscosity as the surrounding medium can exhibit a breakup length greater than that observed for water(Fig. 5). The breakup length starts to grow when the concentration of glycerine in water is above $65 \%$, which in terms of Ohnesorge number is approximately 0.2 . We report that the occurrence of satellite droplets may be supresses if the viscosity of the
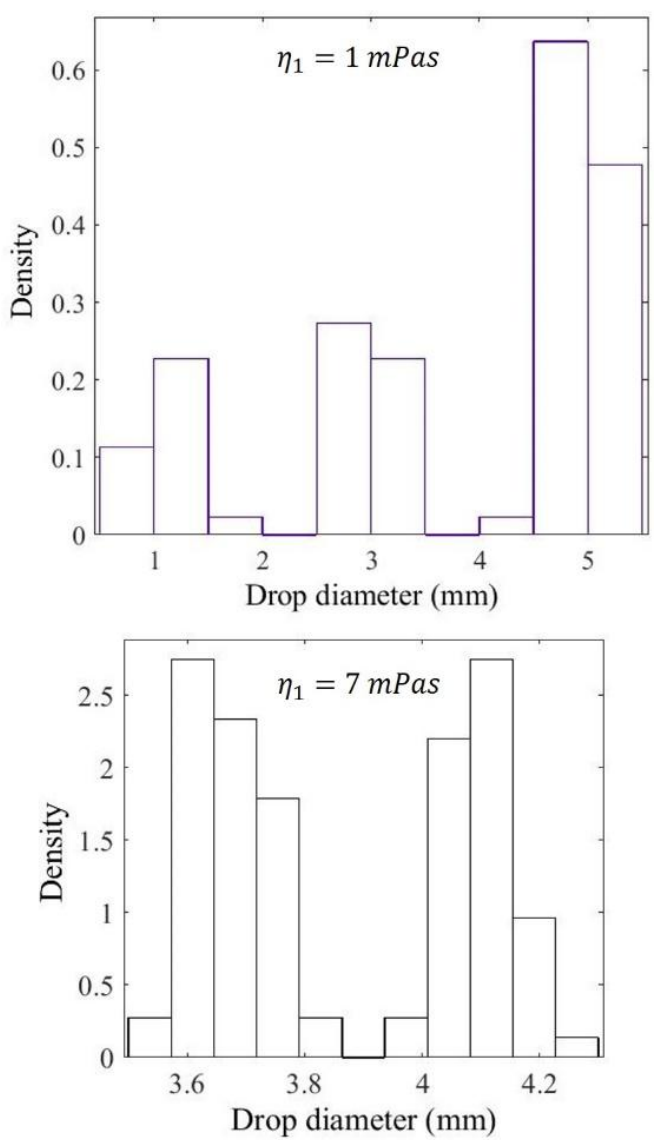

Fig. 4 The influence of viscosity on drop diameter, taking into account both the main drop diameter and the satellite droplets. 
A comparison between deionized water (upper graph) and a $50 \%$ glycerin in water mixture with a viscosity of $7 \mathrm{mPas}$.

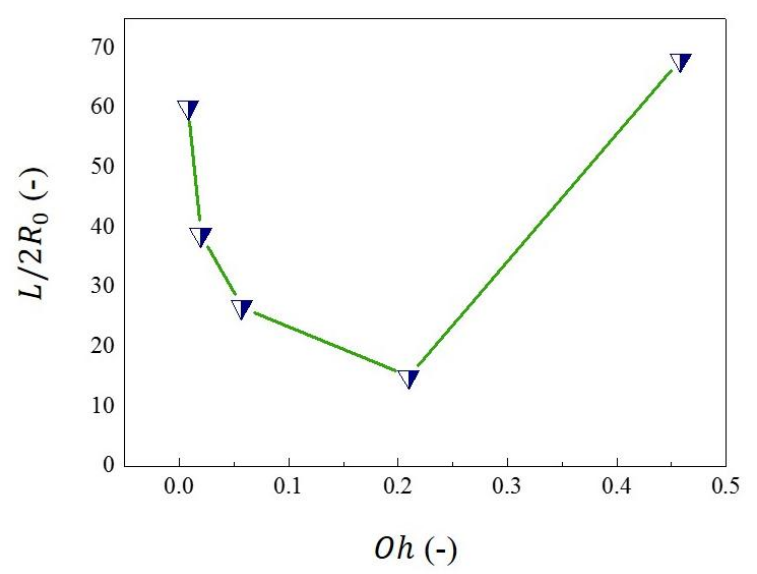

Fig. 5 The dependence of the nondimensional breakup length on Ohnesorge number, $O h=\eta_{1} / \sqrt{\left(\rho_{1} R_{0} \sigma\right)}$. The graph shows a critical point above which the breakup length starts to grow when the viscosity of the injected fluid is increased.

injected fluid is between $7 \mathrm{mPas}$ and $25 \mathrm{mPas}$, the latter representing the viscosity for $65 \%$ glycerine in water.

\section{Conclusions}

In this article, we study the unforced Rayleigh instability of a liquid jet surrounded by another viscous fluid, the resulting system being in an immiscible state. A viscous outer fluid has a direct effect on the dispersion relation relating the growth rate and the wave number, decreasing the value of the most unstable growth rate. From an experimental point of view the second advantage of an external medium is a decrease in the time scales associated with the phenomenon. This offers a way to probe drop detachment and pinch-off without the use of expensive cameras.

Referring to jet breakup, increasing the viscosity of the inner fluid has a decreasing effect on the breakup length. This tendency is not monotonic, above $O h$ numbers greater than 0.2 , the breakup length starts increasing. The second effect of increasing viscosity is a decrease in the main drop diameter and an increase of the satellite drop diameter.The main drop diameter also decreases when the inner radius of the nozzle is decreased. We observe that just by increasing the concentration of glycerine in water the satellite droplets could potentially be eliminated.

This work was partially supported by the grant of the Romanian National Authority for Scientific Research, CNCS, UEFISCDI, PHANTOM - Passive flow control for heat and mass transfer enhancement of impinging jets PN-III-P4-IDPCE-2016-0758 and by the grant of the Romanian space agency ROSA, QUEST - Advanced air diffusion system of the crew quarters for the ISS and deep space habitation systems, STAR-CDI-C3-2016-577.

\section{References}

1. S. Lin,Breakup of liquid sheets and jets, Cambridge University Press, (2003)

2. C. Clasen, P.,Phillips, L. Palangetic, J. Vermant, AIChE J.00, (2011)

3. N.Ashgriz, Handbook of atomization and sprays: theory and applications, Springer Science \& Business Media, (2011)

4. B. Keshavarz et al.,J.N.N.F.M.222, (2015)

5. E. Greiciunas, J.R61467, (2017)

6. A. Myshkis, Low-gravity fluid mechanics,SpringerVerlag, (2012)

7. L. Rayleigh,Proc. Math. Soc, 1.1 (1878)

8. J.Eggers, E. Villermeux, Physics of liquidjets, Reports on progress in physics71.3, (2008)

9. S. Tomotika,Proc. R. Soc. Lond. A, 150 (1935)

10. A. Kalaaji, $\quad$ B. Lopez, P. Attane, A. SoucemarianadinPhys. Fluids 15 9, (2008) 